\title{
ESTRATÉGIAS DE CUIDADOS À FAMÍLIA NO MOMENTO DA PERDA, MORTE ENCEFÁLICA E DOAÇÃO DE ÓRGÃOS, ELABORADAS A PARTIR DE UMA REVISÃO DE LITERATURA DE FATORES QUE LEVAM A FAMÍLIA À RECUSA PARA A DOAÇÃO.
}

\author{
Strategies for family care at the moment of the loss, brain death and organ donation: created from a \\ literature review on main factors that lead to the family refusal to donate organs
}

Neide da Silva Knihs', Bartira de Aguiar Roza², Janine Schirmer²

\begin{abstract}
RESUMO
Objetivo: Identificar os principais fatores que levam a família à recusa para doação de órgãos e assim desenvolver estratégias para amenizar essa recusa. Método: Trata-se de uma revisão de literatura; a busca dos estudos foi por meio de palavras-chaves: doação de órgãos, família, tomada de decisão e transplante de órgãos, no Google, Caps, lilacs, scielo. e MedLine, entre os anos de 2004 a 2008 . Foram encontrados 35 estudos, dos quais 11 foram excluídos por não estarem diretamente relacionados à família e à doação de órgãos. Resultados: Dos 24 estudos avaliados, sete foram dissertações e teses e 17 estavam diretamente relacionados com a recusa da família para doação de órgãos. Após leitura e análise dos estudos, foi possível identificar as principais causas da recusa familiar: falta de acolhimento, não compreensão de Morte Encefálica (ME), insatisfação com o atendimento, falta de comunicação e desconfiança com relação ao processo e medo do comércio dos órgãos. Através das informações obtidas nos estudos e da vivência profissional da autora foram elaboradas as estratégias de cuidado à família nesse processo.
\end{abstract}

Descritores: Doação de Órgãos; Família; Tomada de Decisões; Transplante de Órgãos.

Instituição:

${ }^{1}$ Departamento de Transplante - Sociedade Divina Providencia Hospital Santa Isabel, Blumenau-SC, Brasil

${ }^{2}$ Departamento de Enfermagem da Escola Paulista de Enfermagem - Universidade Federal de São Paulo (UNIFESP), São Paulo-SP, Brasil

\section{Correspondência:}

Neide da Silva Knihs

Rua Dr. Olimpio de Souza Santiago, 81 Ap. 303, CEP 88354-310, Brusque-SC, Brasil Fone: (47) 3350-5596

E-mail: ajknihs@gmail.com

Recebido em: 25.05 .2010
Aceito em: 25.06.2010

\section{INTRODUÇÃO}

No Brasil, com a mudança da legislação a partir de 2001, a Lei $\mathrm{n}^{\mathrm{o}} 10211$, passa a atribuir à família a tomada de decisão em relação à doação de órgãos e tecidos de seu familiar assumindo, assim, a responsabilidade e o compromisso de decidir se autoriza ou não a doação. ${ }^{1}$

A recusa familiar representa uma das principais dificuldades na efetivação da doação de órgãos no Brasil. Essa taxa varia entre 30\% a $70 \%$ nas regiões menos desenvolvidas do país. Um dos principais obstáculos para a realização de um maior número de transplantes é o índice de recusa familiar. ${ }^{2-6}$

Na maioria das vezes, a entrevista para doação ocorre logo após a comunicação do óbito à família, devido à instabilidade do potencial doador. Não é dado a essas pessoas um tempo para assimilar a morte, menos ainda para decidir sobre a doação de órgãos. O tempo torna-se insuficiente para aceitar a perda e reconhecer a morte, levando-se a índices significativos de recusa familiar., ${ }^{4,6-8}$

\section{MÉTODO}

Para a abordagem do tema proposto, foi utilizada primordialmente a experiência profissional da autora, por atuar diretamente na entrevista familiar para doação de órgãos durante 11 anos. A busca 
de estudos deu-se por meio das palavras-chave: doação de órgãos, família e doação, transplante de órgãos, Google, Caps, Lilacs, Scielo e MedLine, compreendendo os anos de 2004 a 2008. Foram encontrados 35 estudos; desses, 11 foram excluídos por abordarem somente o tema transplante e doação de órgãos, não tendo relação com família e doação de órgãos.

\section{RESULTADOS}

Dos 24 estudos avaliados, sete foram dissertações e teses e 17 estavam diretamente relacionados à recusa familiar, ou seja, os motivos que levaram a família a não autorizar a doação. Em 2008 foram encontrados quatro estudos; 2007, seis; 2006, cinco; 2005, seis; 2004, três estudos. Após leitura e avaliação dos resultados de cada um, foi possível identificar que entre as principais causas de recusa familiar para doação de órgãos estão: insatisfação com o atendimento prestado durante a hospitalização, falta de acolhimento e vínculo dos profissionais da saúde com as famílias durante a hospitalização e no processo de doação de órgãos. A comunicação inadequada sobre o quadro clínico de seu familiar, desconfiança na assistência e medo do comércio de órgãos e não entendimento da morte encefálica (ME).

\section{DISCUSSÃO}

A morte de um familiar muda completamente o modo de ser da família, altera a dinâmica, as relações de grupo, significando ruptura da vida e desorganização do mundo. Essas pessoas ficam abaladas, podendo até mesmo apresentar alterações na cognição e na tomada de decisões.

Essas alterações tendem a ser potencializadas quando a perda é acometida por uma causa aguda, e a ME, na maioria das vezes, ocorre por causas agudas e traumáticas.

Quando ocorre a entrevista para doação de órgãos, a família encontra-se em circunstância de dor, sofrimento e angústia diante da perda de um ente querido. $\mathrm{O}$ enfrentamento da morte, associado à dificuldade em decidir sobre a doação, são situações impostas à família logo após a comunicação do óbito, tomando dessas pessoas o direito de chorar a perda do familiar e agredindo física, emocionalmente e eticamente sua privacidade e seus direitos.

As famílias revelam a desumanização, inexistência de acolhimento pelo profissional e pelo serviço de saúde. As famílias relatam problemas no primeiro atendimento e a frieza da equipe de saúde para com eles. ${ }^{4,7-10}$

A falta de acolhimento e vínculo com as famílias, como também as informações e desinformações, geram nessas pessoas insegurança e medo. A falta de atenção nesse processo revelou-se um desestímulo à autorização da doação de órgãos. A dificuldade de acesso às informações, a baixa qualidade destas, bem como a sua contradição, produziram nas famílias um sentido de abandono, desatenção e não compreensão do diagnóstico de morte encefálica. ${ }^{7-9,11}$

Os estudos revelam que $73.9 \%$ das famílias que tiveram seu familiar em ME esclareceram dúvidas em relação à doação, ao passo que $26.1 \%$ não tiveram oportunidade de tirar dúvidas sobre o diagnóstico de ME. 66,7\% não apresentaram dúvidas em relação a esse diagnóstico, enquanto 33,3\% tiveram dúvidas. A maioria dos familiares apresenta compreensão inadequada a respeito do diagnóstico de $\mathrm{ME}$ ou não compreende as informações recebidas e alguns manifestam dúvidas se o familiar estava realmente morto por ocasião da retirada dos órgãos., ${ }^{912-14}$

Em outro estudo realizado nos Estados Unidos, 28.3\% dos membros da família não são capazes de dar corretamente a definição de morte encefálica pela falta de compreensão e entendimento sobre o que acontecia com seu familiar, mesmo após alguns meses do óbito. ${ }^{13}$ É preciso esclarecer previamente na entrevista para solicitação de órgãos à família, o que é ME e quais são os métodos de diagnóstico, através de uma linguagem acessível à compreensão, de forma a garantir-lhes uma escolha consciente e segura. A família fica chocada ao receber a informação do diagnóstico de ME quando não há esclarecimento prévio do quadro clínico., ${ }^{9,12,15-16}$

A família necessita de tempo para assimilar a morte e, principalmente, do respeito moral para com seus sentimentos e sua dor. Cada família tem seu tempo, e cada pessoa, suas próprias características para compreender a ME e aceitar a perda. A perda de um familiar não é apenas a perda de um ente querido, mas de um pai, de uma mãe, de um irmão, enfim, de alguém que até então tinha sonhos, planos objetivos de vida e tinha a morte como algo distante.,17-18

O desconhecimento e/ou não aceitação da ME é compreensível, uma vez que, classicamente, a morte era definida como a cessação irreversível das funções cardíaca e respiratória, o que gera resistência não somente na população, mas também entre profissionais de saúde.

Dentre as dificuldades de aceitar a morte, está o entendimento equivocado sobre a definição de $\mathrm{ME}$ e a dúvida sobre a morte real do familiar que parece vivo, porque seu coração ainda bate e seu corpo é mantido artificialmente por aparelhos. Isso cria insegurança e gera conflito entre os familiares acerca da decisão de consentir a doação, porque a maioria tem o sentimento de perda muito recente. O não entendimento da $\mathrm{ME}$ foi um dos fatores que mais influenciou na recusa para a doação das famílias que participaram dos estudos. , $, 9,11,19-20^{-1}$

Alguns estudos revelaram a descrença das famílias em relação à capacidade dos médicos de realizarem adequadamente o diagnóstico de ME. Muitas delas externaram preocupação referente à possibilidade de morte "provocada" ou "adiada", com o único intuito de obter os órgãos para transplante. A ME é compreendida por muitas famílias como a morte do cérebro e não do restante do corpo. A família sente como se estivesse desligando os aparelhos, se autorizar a doação. ${ }^{10,12,21-22}$

A desconfiança na assistência prestada e o medo do comércio de órgãos geram dúvida, insegurança e a opção pela recusa familiar. A falta de sensibilidade da equipe pode despertar na família o interesse do profissional somente pelos órgãos, gerando a falta de crédito dessas pessoas. ${ }^{13,23-24}$

A família precisa ser cuidada, orientada e acompanhada desde o 
momento em que seu familiar é recebido no Serviço Emergência (SE), independente desta ser ou não entrevistada para doação. Ela percebe quando o profissional aproxima-se somente com o objetivo da doação. É preciso criar com ela uma relação de confiança, compartilhando as informações, levando em consideração seu pouco conhecimento e respeitando a diversidade cultural, religiosa e ética de cada uma. .,8,12,20-21 $^{2}$

A confiança aparece como elemento norteador desse processo. A transparência nas relações da equipe para com as famílias é fator indiscutível na tomada de decisão para a doação. ${ }^{10,12,21-22 .}$

\section{Reflexões}

A literatura referencia a fragilidade da família e a necessidade de cuidado para com ela desde o primeiro contato na hospitalização; essa assistência e esse cuidado devem perdurar durante todo o processo e não somente no momento da entrevista para a doação. Assim, revela-se a importância do cuidado centrado na família, que tem como foco a promoção da relação de ajuda, parceria, articulação e responsabilidade da equipe para com ela.

Frente à revisão desses estudos aliado à vivência da autora durante 11 anos em entrevista para doação, nos pressupostos do interacionismo simbólico e do cuidado centrado na família, foram elaboradas algumas estratégias de cuidado às famílias no momento da perda, morte encefálica e doação de órgãos. Essas estratégias visam uma mesma postura e uma mesma linguagem da equipe interdisciplinar nesse processo.

O interacionismo simbólico é uma teoria em que o significado é o conceito central e são construídas ações individuais e coletivas partindo da interação entre as pessoas, que, definindo situações, agem no contexto social ao qual pertencem. Os pressupostos do cuidado centrado na família visam envolvê-las plenamente no processo de hospitalização, por meio de uma abordagem que é respeito, participação, ética, dignidade e solidariedade. ${ }^{25,26}$

\section{Apresentando as Estratégias:}

Acolhimento a todas as famílias desde o serviço de emergência, perdurando durante toda internação do familiar, no processo de perda até a liberação do corpo. Desde o primeiro contato com a família, o profissional deve estabelecer uma relação de ajuda, ouvir e se colocar à disposição para auxiliá-la nas condutas e decisões que precisam ser tomadas.

Fazer uso de linguagem simples (respeitando o conhecimento da família), procurando não usar termos técnicos e, se for preciso fazer uso dos mesmos, estes devem ser desmistificados de maneira que a família compreenda o que está acontecendo com seu familiar. Usar frases curtas e claras, surgindo dessa interação uma relação de confiança.

Informar a família desde o momento da internação sobre tudo o que está acontecendo (diagnóstico, prognóstico, gravidade), comunicando a estes as condutas que estão sendo tomadas e os exames que foram realizados. Todas as informações deverão ser simples e claras.

Interagir com a família a cada contato. No momento em que ocorrer a abertura do protocolo de ME, a família deve ser informada imediatamente. O profissional deve procurar saber o que ela sabe sobre ME e após informá-la, deverá colocar-se à disposição para dúvidas que ela ainda possa ter.

Por meio da interação (profissional habilitado), buscar conhecer a estrutura familiar, a realidade da família e a posição social que o potencial doador exercia dentro desse sistema.

Por meio da interação junto à família e em local adequado, a ME deve ser comunicada por um médico. Após a comunicação da má noticia, a família deve ser acolhida, cuidada, ouvida, enfim, deve-se partilhar esse momento com a família e estabelecer a relação de ajuda. O diagnóstico deve ser dado tendo todos os exames em mãos, e que o médico deve utilizar linguagem simples, respeitando o conhecimento empírico dos familiares. A ME deve ser desmistificada (através do auxílio de desenhos, imagens, figuras e exames) favorecendo, assim, a compreensão da morte de seu familiar.

Dar um tempo para a família chorar a perda e assimilar a morte (durante esse tempo deverá ser acolhida, acompanhada e cuidada); caso o potencial doador esteja instável, não há possibilidade dessa estratégia.

Preparar o ambiente para sensibilizar a família sobre a doação, sendo que esse deve ser calmo, tranqüilo e sem interferência de outras pessoas. A família somente deverá ser sensibilizada após a equipe interdisciplinar perceber que ela consegue ouvir e compreender que seu familiar está MORTO.

OBSERVAÇÃO: Os profissionais que irão sensibilizar a família sobre a doação deverão ter afinidade com o tema MORTE e família, além da capacidade de conduzir a SENSIBILIZAÇÃO;

Compaixão para com essas pessoas no momento de falar sobre doação. O profissional deve interagir com a dor dessa família e procurar seguir a fala sobre doação de maneira clara, simples, explicando todos os detalhes do processo de doação de órgãos, bem como da liberação do corpo. Todas as dúvidas deverão ser sanadas. Não poderá haver por parte da família nenhuma dúvida ou desconfiança com relação ao processo.

\section{Considerações Finais}

Por meio da revisão e análise dos estudos, foi possível perceber a necessidade de acompanhamento, cuidado e atenção às famílias no momento da perda, morte encefálica e doação de órgãos, bem como a importância de respeitar o tempo da família nesse processo, lembrando que cada uma delas é única e não há como estabelecer um tempo mínimo necessário para que elas possam assumir a morte de seu ente querido e a equipe de saúde possa fazer a entrevista para doação. Os estudos revelam a importância do cuidado, da relação de ajuda às famílias, revelando a essência das estratégias nesse processo, repercutindo na relação de confiança e parceria, oportunizando a essas pessoas a participação no processo de hospitalização e tornando o momento da decisão para doação de órgãos menos agressiva, tanto para a família quanto para o profissional da saúde. 


\section{ABSTRACT}

Purpose: To identify the main factors that lead to the family refusal to donate organs, and therefore to develop strategies to decrease such refusal. Method: It is a literature review. The search for these studies was performed looking for the keywords: organ donation, family, decision making and organ transplantation on Google, Caps, lilacs, scielo and Medline between 2004 and 2008 . It was found 35 studies. 11 studies were excluded since they were not related to the family and organ donation. Results: from 24 studies assessed, seven were dissertations and theses, 17 studies were related to the family refusal to the organ donation. After reading and analyzing, it was possible to identify the major causes to the family refusal, such as: the unwelcomed, misunderstanding on Brain Death (BD), dissatisfaction with the attendance, lack of communication, and lack of confidence in the process, and fear of commerce of human's organs. It was developed strategies for the family care in this process by obtaining information in studies and the author's professional living.

Keywords: Organ Donation; Family; Decision Making; Organ Transplantation.

\section{REFERÊNCIAS}

1. Lei que Altera dispositivo sobre a lei 9434 , de 04 de fevereiro de 1997, que dispõe sobre a remoção de órgãos e tecidos e partes do corpo para fins de transplantes e tratamento, Pub. Diário Oficial da União, N. 10.211(Março 23, 2001)

2. Garcia VD. Situação Atual dos Transplantes no Brasil. Hospital Santa Isabel [Power Point, Apresentação Oral.].Curso de Pó-graduação Lato Sensu em doação de órgãos Univali e Hospital Santa Isabel; 2008.

3. Moraes ELde. A recusa familiar no processo de doação de órgãos e tecidos para transplante (Dissertação). São Paulo: Escola de enfermagem, Universidade Federal de São Paulo: São Paulo; 2007

4. Roza BA. Efeitos do processo de doação de órgãos e tecidos em familiares intencionalidade de uma nova doação (Tese). Escola Paulista de Medicina, Universidade Federal de São Paulo: São Paulo; 2005

5. Santos MJ, Massarolo MCKB. Processo de doação de órgãos: percepção de familiares de doadores cadáveres. Rev. Latino-am. enfermagem. 2005;13(3):382-7.

6. Registro Brasileiro deTransplantes. Estatística 2008, Associação Brasileira de Transplante de Órgãos. [atualizada em janeiro de 2009] Disponível em http://www. abto.com.br- fevereiro 2009.

7. Bousso RS. Um tempo para chorar: a família dando sentido à morte prematura do filho (tese livre-docência). Escola de Enfermagem, Universidade de São Paulo: São Paulo; 2006

8- Daibert MC Recusa Familiar para Doação de Órgãos Na central de notificação, captação e distribuição de órgãos-CNCDO Regional Zona da Mata/Minas Gerais (Dissertação). Faculdade de Serviço Social da Universidade Federal de Juiz de Fora: Minas gerais; 2006.

9. Santiago C. Cross- Cultural Donation and Donation Interview, transplantation Proceedings. 2008; 40: 2882-2

10. Rodrigues CMF, Rech TH. Entrevista familiar e consentimento. Revista Brasileira Terapia Intensiva. 2007;19 (1):85-9.

11. Sque M, LongT, Payne S. organ Donation Key factores influencing families decision making. Transplant.Proc. 2005;37(2):543-46.

12. Sadala MLA. Doação de órgãos: a experiência de enfermeiros, médicos e familiares de doadores (Tese). São Paulo: Faculdade de Medicina de Botucatu, Universidade Estadual Paulista: São Paulo; 2004.
13. Rithalia C. Mcdaid C. Suekarran S. Norman G, Myer A S. A systematic review of presumed consent systems for deceased organ donation. American Journal of Transplantation. 2007;7:1778-87

14. Frutos MA, Blanca MJ, Mansillo JJ, Rando B, Ruiz P, Guerrero F. Organ donation: comparison of donating and nondonating families. Transplant. Proceedings. 2005; 37(3):1557-9.

15. Lang F, Quill 1 T. Making decisions with families at the end of life. American Academy of Family Physicians. 2004;70(4):719-23.

16. Padrão MB, Lima AA, Moraes EL. Fatores que influenciam a recusa familiar no processo de doação de órgãos e tecidos para transplantes. J BrasTranspl. 2004;7:137-40.

17. Miziraji R, Alvarez I, Palacios RI, Farjado C, Berreios C, Morales F. Organ donation in Latin America. Trasplant Proceedings. 2007;39(2):333-5.

18. Fuhr M. O significado do processo de doação de órgãos para familiares doadores (Dissertação) Universidade Federal de Santa Catarina: Florianópolis; 2006..

19. Siminoff L, Mercer MB, Graham G, Burant C. The reasons families donate organs for transplantation: implications for policy and practice. J Trauma. 2007; 62(4):969-78.

20. Alencar SCS. de Doação de órgãos e tecidos: a vivência dos familiares de crianças e adolescentes doadores (Dissertação). Universidade Federal do Paraná: Curitiba; 2006.

21. Bousso RS.O Processo de decisão familiar na doação de órgãos do filho: Uma teoria substantiva. Texto Contexto Enferm. 2008;17(1):45-54.

22. Barber K, Favey S, Hamilton C, Collet D, Rudge C. Potencial for organ donation in the united Kingdon: audito of intensive care records. BMJ. 2006;332(7550):1105-6.

23. Rodrigues JR, Cornell DL, Howard RJ. The Instability of Organ Donation Decisions by Next-of-Kin and Factors That Predict It. American Journal of Transplantation. 2008;8:2661-7

24. Karaman EM, Dermican A, Dalgic A. Potential organ donor concept is developing in emergency departments: Gazi University Hospital experience. Transplant Proc. 2008;40(1):39-41.

25- . interativo em enfermagem. Rev Esc Enferm USP. 2005; 39 (1):103-8,

26. Haire SO, Blacford JC. Nurses moral agency in negotiating parental participation in care. International of Journal of Nursing Practice. 2005;11:250-6. 